論 文

\title{
入出力特性を補間する線形離散時間システムの パラメトリゼーション*
}

\author{
A Parametrization of Linear Discrete-Time Systems \\ Interpolating Input-Output Characteristics*
}

Kazumi HoRIGUCHI**

\begin{abstract}
Problems of finding linear systems which are consistent with given input-output characteristics are important in system approximation, model reduction and system identification. A conventional problem among them is the partial realization problem. In this paper, we consider a problem of finding MIMO linear discrete-time systems interpolating given input-output characteristics. The input-output characteristics are given by coefficients of the Taylor series of the transfer function at some complex points in the unit circle. Thus our problem is a generalization of the partial realization problem. Here, we provide a parametrization of all the interpolating systems with a norm constraint and a parametrization of all the stable interpolating systems.
\end{abstract}

\section{1.はじめに}

与えられた入出力特性に適合するような線形システム を求めることは, システムの近似, 低次元化, および, シ ステム同定における重要な問題である。このような問題 のうち, 従来から知られてきたものの一つに部分実現問 題がある1),2).

本稿では，与えられた入出力特性を補間する多入力多 出力線形離散時間システム—以降, 補間システムと呼 ぶ—を求める問題を考察する. 入出力特性は, 単位円内 のいくつかの複素周波数点における伝達関数のテイラー 展開係数として与えられる。したがって，ここで考察す る問題は部分実現問題の一般化になっている.

部分実現問題も含めて, 補間システムを求める問題に ついては，十分な結果が報告されてきた3),4)。しかし，補 間システムにある制約を課したとき，その制約を満たすよ うな補間システムを求める問題はそれほど簡単ではない. 本稿では, ノルム制約のある補間システム, および, 安 定な補間システムを求める問題を考察する。これらの問題 について, いくつかの結果が報告されているが3),5)-11),

* 原稿受付 1997 年 4 月 4 日

** 近畿大学 生物理工学部 Faculty of Biology Oriented Science and Technology, Kinki University; 930 Nishimitani, Uchita, Naga-gun Wakayama 649-6493, JAPAN Key Words: input-output characteristics, interpolation, linear discrete-time system, parametrization.
ここでは, 新しいアプローチに基づいて, 次の結果を提 供している。(i) ノルム制約のあるすべての補間システ ムのパラメトリゼーション。これは従来の結果を包含し た新しい表現である。（ii）安定なすべての補間システ ムのパラメトリゼーション.これは従来の結果と一致し, 具体的な構成手順を与えた表現である.

以下では次の記法を用いる。

$$
\begin{aligned}
& \zeta: \text { 遅延要素 }\left(=z^{-1}\right) \\
& C: \text { 複素数体 } \\
& C^{m \times r}: C \text { 上の } m \times r \text { 行列の集合 } \\
& R H_{\infty}^{m \times r}=\{F(\zeta) \mid F(\zeta) \text { は }|\zeta| \leq 1 \text { に極を } \\
& \quad \text { 持たない } m \times r \text { 有理関数行列 }\} \\
& \bar{B} R H_{\infty}^{m \times r}=\left\{F(\zeta) \mid F(\zeta) \in R H_{\infty}^{m \times r}\right. \text { かつ } \\
& B R H_{\infty}^{m \times r}=\left\{F(\zeta) \mid F(\zeta) \in \bar{B} R H_{\infty}^{m \times r}\right. \text { かつ } \\
& \left.\quad\|F(\zeta)\|_{\infty} \leq 1\right\} \\
& F^{\sim}(\zeta)=F^{*}\left(\zeta^{-1}\right) \quad\left({ }^{*}: \text { 行列の共役転置 }\right) \\
& \operatorname{deg} F(\zeta): F(\zeta) \text { のマクミラン次数 }
\end{aligned}
$$

\section{2. 問題の定式化}

$r$ 入力 $m$ 出力の線形離散時間システムの伝達関数を $H(\zeta)$ と書く. 入出力特性 


$$
\begin{aligned}
\zeta_{i}, H_{i j}^{o} \quad\left(\zeta_{i} \in C,\left|\zeta_{i}\right|<1, H_{i j}^{o} \in C^{m \times r},\right. \\
\left.i=1,2, \ldots, p, j=0,1, \ldots, q_{i}-1\right)
\end{aligned}
$$

が与えられたとき，伝達関数が

$$
\begin{array}{r}
H(\zeta)=\sum_{j=0}^{q_{i}-1} H_{i j}^{o}\left(\zeta-\zeta_{i}\right)^{j}+O\left(\left(\zeta-\zeta_{i}\right)^{q_{i}}\right) \\
(i=1,2, \ldots, p)
\end{array}
$$

を満たすシステムを考える。

(2) 式は, $H(\zeta)$ を複素周波数点 $\zeta_{i}$ においてテイラー展 開したとき，展開係数のはじめから $q_{i}$ 個が与えられた值 に一致する条件を表している．同式はまた， $H(\zeta)$ が複素 周波数点 $\zeta_{i}$ において入出力特性 $H_{i j}^{o}\left(j=0,1, \ldots, q_{i}-1\right)$ を補間する条件と解釈できる。ここで， $\zeta_{i}$ を補間点, $q_{i}$ を饻における重複度と呼び, 伝達関数が $(2)$ 式を満たす システムを, 入出力特性 (1) 式を補間するシステム, あ るいは単に，補間システムと呼ぶ.

本稿では, 伝達関数の $H_{\infty}$ ノルムがある值以下である 補間システムを求める問題, および, 安定な補間システ 厶を求める問題を考える. 伝達関数の $H_{\infty}$ ノルムがある 值 $(\gamma)$ 以下である条件は

$$
\gamma^{-1} H(\zeta) \in \bar{B} R H_{\infty}^{m \times r} \quad(\gamma>0)
$$

と表される．システムが安定である条件は

$$
H(\zeta) \in R H_{\infty}^{m \times r}
$$

\section{と表される。}

これより, 本稿で考察する問題は次に定式化される.

【問題 1】入出力特性 (1) 式が与えられたとき，(2), (3) 式を満たすシステムを求めよ.

【問題 2】 入出力特性 (1) 式が与えられたとき，(2), (4) 式を満たすシステムを求めよ。

入出力特性 (1) 式で, $p=1, \zeta_{1}=0$ としたとき, $H_{1 j}^{o}$ は システムの Markov パラメータを表し, これらの補間シ ステムを求める問題は部分実現問題へと帰着される1),2).

部分実現問題も含めて, 補間システムを求める問題に ついては，十分な結果が得られている．参考文献3)では， すべての補間システムのパラメトリゼーションが与えら れている。 また, 参考文献 4) では, 参考文献 3) の結果 にマクミラン次数の考察が加えられ, 最小マクミラン次 数を持つすべての補間システムのパラメトリゼーション が与えられている.

一方，(3)式または (4) 式を満たす補間システムを求め る問題はそれほど簡単ではない.

問題 1 は $H_{\infty}$ 制御問題と密接な関係があり,この種 の補間問題が $H_{\infty}$ 制御問題に帰着されること5), 逆に,
$H_{\infty}$ 制御問題がこの種の補間問題に州着されること汭が それぞれ指摘されている. $H_{\infty}$ 制御問題の解法とも関連 して，ノルム制約のある補間問題の解法がいくつか提案 されてきた。それらは拡張 Nevanlinna アルゴリズムに よる逐次解法 5)-7) と, 状態空間による解法3),8)-10)に大 別される. 後者は前者に比べて, 補間点の重複への対応 が容易である点, 解が explicitにパラメトライズされる 点で優れている.

問題 1で, (3) 式の条件に代えて

$$
\gamma^{-1} H(\zeta) \in B R H_{\infty}^{m \times r} \quad(\gamma>0)
$$

の条件を考えた場合，問題は扱いやすく，状態空間法に 基づいて (5) 式を満たすすべての補間システムのパラメ トリゼーションが提供されている3),10). (3) 式を満たす すべての補間システムのパラメトリゼーションは, 連続 時間システムに対して与えられているが9), これは(5)式 の条件を考えた場合と同様の簡潔な表現ではない.

問題 2については, SISO システムに対する安定な補 間システムの一構成法が提案されている11)。また, 参考 文献 3) では，安定なすべての補間システムのパラメトリ ゼーションが提供されているが, その具体的な構成手順 には言及されていない．

本稿では，まず 3.で，状態空間による新しいアプロー チに基づき，(3) 式を満たすすべての補間システムのパ ラメトリゼーションを提供する。これは，(5)式の条件を 考えた場合の結果 3 ),10)を包含した新しい表現となってい る. 次に 4.で, 3.の展開に基づき，(4)式を満たすすべて の補間システムのパラメトリゼーションを提供する。こ れは従来の結果 ${ }^{3)}$ と一致し, 具体的な構成手順を与えた 表現となっている.

\section{3. ノルム制約のある補間システム}

ここでは, 問題 1 の解法を展開する.

はじめに，(2),(3) 式を状態空間表現することを考え る. $H(\zeta)$ が $(2)$ 式を満たすことは, $H(\zeta)$ の適当な実現 $(A, B, C, D)\left(A \in C^{n \times n}\right)$ に対し, $Q \in C^{n \times q r}$ が存在して

$$
\begin{aligned}
& {\left[\begin{array}{ll}
A & B \\
C & D
\end{array}\right]\left[\begin{array}{c}
Q \Lambda \\
E
\end{array}\right]=\left[\begin{array}{l}
Q \\
F
\end{array}\right]} \\
& \operatorname{det}\left(I-\zeta_{i} A\right) \neq 0 \quad(i=1,2, \ldots, p)
\end{aligned}
$$

が成立することと等価である12)。ここで， $\Lambda, E, F$ はそ れぞれ入出力特性 (1) 式から作られる次の行列である.

$$
\Lambda=\operatorname{diag}\left[\Lambda_{1}, \cdots, \Lambda_{p}\right] \in C^{q r \times q r}
$$


$\Lambda_{i}=\left[\begin{array}{cccc}\zeta_{i} I_{r} & I_{r} & & \bigcirc \\ & \ddots & \ddots & \\ & & \ddots & I_{r} \\ \bigcirc & & & \zeta_{i} I_{r}\end{array}\right] \in C^{q_{i} r \times q_{i} r}$

$E=\left[\begin{array}{lll}E_{1} & \cdots & E_{p}\end{array}\right] \in C^{r \times q r}$

$E_{i}=\left[\begin{array}{llll}I_{r} & 0 & \cdots & 0\end{array}\right] \in C^{r \times q_{i} r}$

$F=\left[\begin{array}{lll}F_{1} & \cdots & F_{p}\end{array}\right] \in C^{m \times q r}$

$F_{i}=\left[\begin{array}{lll}H_{i 0}^{o} & \cdots & H_{i q_{i}-1}^{o}\end{array}\right] \in C^{m \times q_{i} r}$

$I_{r}: r$ 次単位行列, $q=q_{1}+\cdots+q_{p}$

つぎに，(3) 式を考える. $H(\zeta)$ が $(3)$ 式を満たすなら ば，その可到達·可観測な実現 $(A, B, C, D)\left(A \in C^{n \times n}\right)$ に対し, $X>0, C_{2} \in C^{r \times n}, D_{2} \in C^{r \times r}$ が存在して

$$
\begin{aligned}
& A^{*} X A+\gamma^{-2} C^{*} C+C_{2}^{*} C_{2}=X \\
& A^{*} X B+\gamma^{-2} C^{*} D+C_{2}^{*} D_{2}=0 \\
& B^{*} X B+\gamma^{-2} D^{*} D+D_{2}^{*} D_{2}=I
\end{aligned}
$$

が成立する ${ }^{13)}$.ここで, $\left(A, B, C, C_{2}\right)$ に適当な相似変換 を施せば，上式は

$$
\begin{aligned}
& {\left[\begin{array}{cc}
A & B \\
\bar{C} & \bar{D}
\end{array}\right]^{*}\left[\begin{array}{cc}
A & B \\
\bar{C} & \bar{D}
\end{array}\right]=I} \\
& \bar{C}=\left[\begin{array}{c}
\gamma^{-1} C \\
C_{2}
\end{array}\right], \quad \bar{D}=\left[\begin{array}{c}
\gamma^{-1} D \\
D_{2}
\end{array}\right]
\end{aligned}
$$

と書き換えられる（相似変換後も同じ記法を用いた）。す なわち, $H(\zeta)$ が $(3)$ 式を満たすならば，その適当な実 現 $(A, B, C, D)$ に対し, $C_{2}, D_{2}$ が存在して (8) 式が成立 する。また, $(A, C)$ は可観測だから, $(A, \bar{C})$ も可観測で ある・

逆に, $H(\zeta)$ の適当な実現 $(A, B, C, D)$ に対し, $C_{2}, D_{2}$ が存在して $(8)$ 式が成り立ち, $(A, \bar{C})$ は可観測とする. このとき, $A^{*} A+\bar{C}^{*} \bar{C}=I$ だら， $A$ は安定行列（固有 值がすべて単位円内に存在する行列 ) である。そこで, $H_{2}(\zeta)=D_{2}+\zeta C_{2}(I-\zeta A)^{-1} B$ としたとき，(8) 式よ り, $\gamma^{-2} H^{\sim}(\zeta) H(\zeta)+H_{2}^{\sim}(\zeta) H_{2}(\zeta)=I$ となるから13), $H(\zeta)$ は $(3)$ 式を満たす.

以上より, $H(\zeta)$ が $(3)$ 式を満たすことは, $H(\zeta)$ の適 当な実現 $(A, B, C, D)$ に対し, $C_{2}, D_{2}$ が存在して (8) 式 が成り立ち， $(A, \bar{C})$ が可観測となることと等価である.

ところで, $\left|\zeta_{i}\right|<1$ だから, (8) 式は (7) 式を含んでい
る.したがって，(2)，(3) 式を状態空間表現すれば，(6), (8) 式となることがわかり, 次の補題が得られた.

【補題 1】次の二つの命題は等価である.

(i) $H(\zeta)$ は (2), (3) 式を満たす.

(ii) $H(\zeta)$ の適当な実現 $(A, B, C, D)\left(A \in C^{n \times n}\right)$ に対 し, $C_{2} \in C^{r \times n}, D_{2} \in C^{r \times r}, Q \in C^{n \times q r}$ が存在し て $(6),(8)$ 式が成り立ち, $(A, \bar{C})$ は可観測となる. ここで, (ii)において, $(A, B)$ の可到達性, $(A, C)$ の可観 測性はいずれも必要とされていないことに注意されたい.

補題 1 より, 問題 1は, “ある $C_{2}, D_{2}, Q$ に対して, (6), $(8)$ 式を満たし, $(A, \bar{C})$ が可観測となるような $H(\zeta)$ の 実現 $(A, B, C, D)\left(A \in C^{n \times n}\right)$ を求めよ.”という問題へ と帰着された. 以下では, このような $(A, B, C, D)$ を求 めることを考える. $A$ の次元は $n$ とする. 上述のとおり, $(A, B, C, D)$ は必ずしも $H(\zeta)$ の最小実現ではないので, $\operatorname{deg} H(\zeta) \leq n$ である.

まず，(2)，(3) 式を満たすシステムの存在条件を導く. $H(\zeta)$ が $(2),(3)$ 式を満たすとする. このとき, $C_{2}, D_{2}, Q$ が存在して (6), (8) 式が成立するが，ここで

$$
\left[\begin{array}{ll}
C_{2} & D_{2}
\end{array}\right]\left[\begin{array}{c}
Q \Lambda \\
E
\end{array}\right]=F_{2}
$$

とおいて，(6)式を

$$
\left[\begin{array}{cc}
A & B \\
\bar{C} & \bar{D}
\end{array}\right]\left[\begin{array}{c}
Q \Lambda \\
E
\end{array}\right]=\left[\begin{array}{c}
Q \\
\bar{F}
\end{array}\right], \quad \bar{F}=\left[\begin{array}{c}
\gamma^{-1} F \\
F_{2}
\end{array}\right]
$$

と書くことにする. (8) 式の左から $\left[\Lambda^{*} Q^{*} E^{*}\right]$ を, 右 から $\left[\begin{array}{c}Q \Lambda \\ E\end{array}\right]$ をそれぞれ乗じて，(10)式を用いると

$$
\begin{aligned}
& \Lambda^{*} P \Lambda-P+E^{*} E-\bar{F}^{*} \bar{F}=0 \\
& P=Q^{*} Q
\end{aligned}
$$

を得る。

(11) 式は $P$ に関する Lyapunov 方程式であり， $\Lambda$ の 定義からその解は一意である14)。よって，(2)，(3) 式を 満たすシステムが存在するならば，(11) 式は半正定解 $P=Q^{*} Q$ を持つ. ただし， $F_{2}$ は入出力特性 (1) 式から 直接計算できないので, $P$ の半正定性は $(2),(3)$ 式を満 たすシステムが存在するための必要条件として実用的で ない.

実際には，(2)，(3) 式を満たすシステムの存在性につ いて次の定理が成立する。

【定理 1】 (2), (3) 式を満たすシステムが存在するた めの必要十分条件は, Lyapunov 方程式

$$
\Lambda^{*} P_{0} \Lambda-P_{0}+E^{*} E-\gamma^{-2} F^{*} F=0
$$


の解 $P_{.0}$ が半正定となることである.

（証明）必要性:上述より，(11) 式の解は $P \geq 0$ である. Lyapunov 方程式 (11) と (12) を比較すると, $-\gamma^{-2} F^{*} F$ $+\bar{F}^{*} \bar{F}=F_{2}^{*} F_{2} \geq 0$ だから, それらの解について $P_{0} \geq P$ となる14).よって, (2), (3) 式を満たすシステムが存在 するならば, $P_{0} \geq P \geq 0$ となり, 必要性が言えた。

十分性： $P_{0} \geq 0$ のとき, $(2),(3)$ 式を満たすシステムを 具体的に構成することにより示す.その構成は定理 $\mathbf{2}$ の 中で行う.

以降, Lyapunov 方程式 (12) の解 $P_{0}$ は半正定と仮定 する. 以下では, (10) 式において $F_{2}=0$ とした次の式

$$
\left[\begin{array}{cc}
A & B \\
\bar{C} & \bar{D}
\end{array}\right]\left[\begin{array}{c}
Q \Lambda \\
E
\end{array}\right]=\left[\begin{array}{c}
Q \\
\bar{F}_{0}
\end{array}\right], \quad \bar{F}_{0}=\left[\begin{array}{c}
\gamma^{-1} F \\
0
\end{array}\right]
$$

を考え, (8), (13) 式を満たす任意の $(A, B, \bar{C}, \bar{D})$ の表現 を求める (補題 2). ついで, この結果に基づいて, (2), (3) 式を満たすすべてのシステムのパラメトリゼーション を与える(定理 2).

Lyapunov 方程式 (12) の半正定解が $\operatorname{rank} P_{0}=n_{0}$ で あるとする. $n_{0}$ は, $(8),(13)$ 式を満たす $A$ の次元の最 小値となる (補題 2 ). このとき, $P_{0}$ のフルランク分解を

$$
P_{0}=Q_{0}^{*} Q_{0} \quad\left(Q_{0} \in C^{n_{0} \times q r}\right)
$$

と書く. (12), (14) 式から

$$
\left[\begin{array}{ll}
\Lambda^{*} Q_{0}^{*} & E^{*}
\end{array}\right]\left[\begin{array}{c}
Q_{0} \Lambda \\
E
\end{array}\right]=\left[\begin{array}{ll}
Q_{0}^{*} & \gamma^{-1} F^{*}
\end{array}\right]\left[\begin{array}{c}
Q_{0} \\
\gamma^{-1} F
\end{array}\right]
$$

となるから, 次の特異值分解 (SVD) が定義できる.

$$
\begin{aligned}
& {\left[\begin{array}{c}
Q_{0} \Lambda \\
E
\end{array}\right]=\left[\begin{array}{ll}
V_{11} & V_{12} \\
V_{21} & V_{22}
\end{array}\right]\left[\begin{array}{cc}
\Sigma & 0 \\
0 & 0
\end{array}\right]\left[\begin{array}{c}
U_{1}^{*} \\
U_{2}^{*}
\end{array}\right]} \\
& {\left[\begin{array}{c}
Q_{0} \\
\gamma^{-1} F
\end{array}\right]=\left[\begin{array}{ll}
W_{11} & W_{12} \\
W_{21} & W_{22}
\end{array}\right]\left[\begin{array}{cc}
\Sigma & 0 \\
0 & 0
\end{array}\right]\left[\begin{array}{c}
U_{1}^{*} \\
U_{2}^{*}
\end{array}\right]} \\
& \operatorname{rank}\left[\begin{array}{c}
Q_{0} \Lambda \\
E
\end{array}\right]=\operatorname{rank}\left[\begin{array}{c}
Q_{0} \\
\gamma^{-1} F
\end{array}\right]=\nu \\
& \Sigma=\operatorname{diag}\left[\begin{array}{lll}
\sigma_{1} & \cdots & \sigma_{\nu}
\end{array}\right], \quad \sigma_{i}>0
\end{aligned}
$$

また，(16)式より，次のSVD が定義される.

$$
\begin{aligned}
& {\left[\begin{array}{c}
Q_{0} \\
\bar{F}_{0}
\end{array}\right]=\left[\begin{array}{ll}
W_{11} & \bar{W}_{12} \\
\bar{W}_{21} & \bar{W}_{22}
\end{array}\right]\left[\begin{array}{cc}
\Sigma & 0 \\
0 & 0
\end{array}\right]\left[\begin{array}{c}
U_{1}^{*} \\
U_{2}^{*}
\end{array}\right]} \\
& \bar{W}_{12}=\left[\begin{array}{ll}
W_{12} & 0_{n_{0} \times r}
\end{array}\right], \quad \bar{W}_{21}=\left[\begin{array}{c}
W_{21} \\
0_{r \times \nu}
\end{array}\right]
\end{aligned}
$$

$$
\bar{W}_{22}=\left[\begin{array}{cc}
W_{22} & 0 \\
0 & I_{r}
\end{array}\right] \quad\left(0_{p \times q}: p \times q \text { 零行列 }\right)
$$

ここで, $\nu \leq \min \left\{n_{0}+r, n_{0}+m\right\}$ であるが, 上式では $\nu<\min \left\{n_{0}+r, n_{0}+m\right\}$ を前提として SVDを記述した. 以下では, この前提のもとで $(2),(3)$ 式を満たす $H(\zeta)$ のパラメトリゼーションを与える.一方, $\nu=n_{0}+r$ ま たは $\nu=n_{0}+m$ の場合， $(2),(3)$ 式を満たす $H(\zeta)$ は一 意に与えられるが，これについては注意 2 で言及する.

これらの定義のもとで, 次の補題が成り立つ.

【補題 2】 $P_{0} \geq 0, \operatorname{rank} P_{0}=n_{0}$ とする.このとき, $(8),(13)$ 式を満たす $(A, B, \bar{C}, \bar{D})\left(A \in C^{n \times n}\right)$ は $n \geq n_{0}$ でなければならず, そのような任意の $(A, B, \bar{C}, \bar{D})$ は

$$
\begin{aligned}
{\left[\begin{array}{c|c}
A_{0} & B_{0} \\
\hline \bar{C}_{0} & \bar{D}_{0}
\end{array}\right]=} & {\left[\begin{array}{c}
W_{11} \\
0 \\
\hline \bar{W}_{21}
\end{array}\right]\left[\begin{array}{ll|l}
V_{11}^{*} & 0 & V_{21}^{*}
\end{array}\right] } \\
& +\left[\begin{array}{cc}
0 & \bar{W}_{12} \\
I & 0 \\
\hline 0 & \bar{W}_{22}
\end{array}\right]\left[\begin{array}{cc}
A_{\theta} & B_{\theta} \\
\bar{C}_{\theta} & \bar{D}_{\theta}
\end{array}\right]\left[\begin{array}{cc|c}
0 & I & 0 \\
V_{12}^{*} & 0 & V_{22}^{*}
\end{array}\right]
\end{aligned}
$$

の相似なシステムとして与えられる.ここで, $\left(A_{\theta}, B_{\theta}\right.$, $\left.\bar{C}_{\theta}, \bar{D}_{\theta}\right)$ は $\left(n_{0}+r-\nu\right)$ 入力 $\left(n_{0}+m-\nu+r\right)$ 出力 $n_{\theta}(=$ $\left.n-n_{0}\right)$ 次のシステムで

$$
\left[\begin{array}{cc}
A_{\theta} & B_{\theta} \\
\bar{C}_{\theta} & \bar{D}_{\theta}
\end{array}\right]^{*}\left[\begin{array}{cc}
A_{\theta} & B_{\theta} \\
\bar{C}_{\theta} & \bar{D}_{\theta}
\end{array}\right]=I
$$

を満たす限り任意である.

また，(18) 式の $\left(A_{0}, B_{0}\right)$ は, $\left(A_{\theta}, B_{\theta}\right)$ が可到達なら ば, 可到達であり, $\left(A_{0}, \bar{C}_{0}\right)$ は, $\left(A_{\theta}, \bar{C}_{\theta}\right)$ が可観測なら ば，可観測である。

(証明) 参考文献 12)の定理 1, 2 と同様に証明できる ので，ここでは概略のみを記す. (13) 式の $Q \in C^{n \times q r}$ よ り作られる $Q^{*} Q$ は Lyapunov 方程式 (12) の解であるか ら, $P_{0}=Q^{*} Q=Q_{0}^{*} Q_{0}$ となる. $Q_{0}$ は行フルランクだか ら，ユニタリ行列 $T$ が存在して $Q=T\left[\begin{array}{c}Q_{0} \\ 0\end{array}\right]$ と書ける. $(A, B, \bar{C}, \bar{D})$ に $T$ を用いて相似変換を施せば, $(13)$ 式は

$$
\left[\begin{array}{cc}
A & B \\
\bar{C} & \bar{D}
\end{array}\right]\left[\begin{array}{c}
Q_{0} \Lambda \\
0 \\
E
\end{array}\right]=\left[\begin{array}{c}
Q_{0} \\
0 \\
\bar{F}_{0}
\end{array}\right], \quad \bar{F}_{0}=\left[\begin{array}{c}
\gamma^{-1} F \\
0
\end{array}\right]
$$

と書き換えられる（相似変換後も同じ記法を用いた）。 そこで，(13) 式に代えて (20) 式を考えればよい. (18) 式のシステムが (8)，(20) 式を満たすこと，逆に，(8), (20) 式を満たすシステムが (18) 式で表されることは, (15), (17)，(19) 式などから確認できる.つぎに，(18) 
式の $\left(A_{0}, B_{0}\right)$ の可到達性と $\left(A_{0}, \bar{C}_{0}\right)$ の可観測性につい てであるが,これらの議論は同様に行えるので, ここで は $\left(A_{0}, \bar{C}_{0}\right)$ の可観測性のみを考える. $\lambda \in C$ が存在し て $\left(\lambda I-A_{0}\right) x=-\bar{C}_{0} x=0$ が成り立ち, $\left(A_{\theta}, \bar{C}_{\theta}\right)$ は可 観測と仮定する。このとき， $A_{0}^{*} A_{0}+\bar{C}_{0}^{*} \bar{C}_{0}=I$ だか， $|\lambda|=1$ である. また, 仮定の式に (18) 式の $A_{0}, \bar{C}_{0}$ を代 入し,$x=\left[\begin{array}{ll}x_{1}^{T} & x_{2}^{T}\end{array}\right]^{T}$ と書いて整理すると

$$
\begin{aligned}
& \left(\lambda W_{11}^{*}-V_{11}^{*}\right) x_{1}=0 \\
& -B_{\theta} V_{12}^{*} x_{1}+\left(\lambda I-A_{\theta}\right) x_{2}=0 \\
& \left(\lambda \bar{W}_{12}^{*}-\bar{D}_{\theta} V_{12}^{*}\right) x_{1}-\bar{C}_{\theta} x_{2}=0
\end{aligned}
$$

を得る. (15), (17), (21) 式より $\left(\lambda I-\Lambda^{*}\right) Q_{0}^{*} x_{1}=0$ とな り, $|\lambda|=1, Q_{0}$ : 行フルランクだから $x_{1}=0$ である。ゆ えに, $(22),(23)$ 式と仮定より $x_{2}=0$ となり, 結局, $x=0$ がいえる.よって, $\left(A_{\theta}, \bar{C}_{\theta}\right)$ が可観測ならば $\left(A_{0}, \bar{C}_{0}\right)$ は 可観測であることが示された.

3.の目標は, 補題 1 を考慮すれば，(6), (8) 式を満た し, $(A, \bar{C})$ が可観測なすべてのシステムをパラメトライ ズすることである. 補題 2 は (8), (13) 式を満たす任意の システムの表現を示している，ところで，(13) 式は (6) 式を含んでいるから，(18) 式は (6)，(8) 式を満たすシス テムの部分集合を与えている．実際には，(18) 式は (6), (8) 式を満たすすべてのシステムの表現を与える. 次の 定理はこのことを示し, 問題 1 の解を提供している.

【定理 2】 $P_{0} \geq 0, \operatorname{rank} P_{0}=n_{0}$ とする.このとき, (2), (3) 式を満たすすべてのシステムは

$$
\begin{aligned}
H(\zeta)=\Sigma_{11}(\zeta)+ & \Sigma_{12}(\zeta) \Theta(\zeta) \\
& \cdot\left\{I-\Sigma_{22}(\zeta) \Theta(\zeta)\right\}^{-1} \Sigma_{21}(\zeta)
\end{aligned}
$$

でパラメトライズされる.ここで, $\Sigma_{i j}(\zeta)$ は

$$
\begin{aligned}
\Sigma(\zeta) & =\left[\begin{array}{cc}
\Sigma_{11}(\zeta) & \Sigma_{12}(\zeta) \\
\Sigma_{21}(\zeta) & \Sigma_{22}(\zeta)
\end{array}\right] \\
& \stackrel{\mathrm{S}}{=}\left[\begin{array}{c|cc}
W_{11} V_{11}^{*} & W_{11} V_{21}^{*} & \gamma^{-1} W_{12} \\
\hline \gamma W_{21} V_{11}^{*} & \gamma W_{21} V_{21}^{*} & W_{22} \\
V_{12}^{*} & V_{22}^{*} & 0
\end{array}\right]
\end{aligned}
$$

である.また, $\Theta(\zeta)$ は

$$
\gamma^{-1} \Theta(\zeta) \in \bar{B} R H_{\infty}^{\left(n_{0}+m-\nu\right) \times\left(n_{0}+r-\nu\right)}
$$

なる任意の関数である.

（証明）(24) 式の $H(\zeta)$ が $(2),(3)$ 式を満たすこと：補 題 1 と $(26)$ 式より, $\Theta(\zeta)$ の適当な実現 $\left(A_{\theta}, B_{\theta}, C_{\theta}, D_{\theta}\right)$ $\left(A_{\theta} \in C^{n_{\theta} \times n_{\theta}}\right)$ に対し, $C_{\theta 2}, D_{\theta 2}$ が存在して, $\bar{C}_{\theta}=$ $\left[\begin{array}{c}\gamma^{-1} C_{\theta} \\ C_{\theta 2}\end{array}\right], \bar{D}_{\theta}=\left[\begin{array}{c}\gamma^{-1} D_{\theta} \\ D_{\theta 2}\end{array}\right]$ とおくとき, (19) 式が成り
立ち, $\left(A_{\theta}, \bar{C}_{\theta}\right)$ は可観測となる。この $\left(A_{\theta}, B_{\theta}, C_{\theta}, D_{\theta}\right)$ を用いて, (24) 式の $H(\zeta)$ の実現の一つは

$$
\begin{aligned}
& {\left[\begin{array}{c|c}
A & B \\
\hline C & D
\end{array}\right]=\left[\begin{array}{c}
W_{11} \\
0 \\
\hline \gamma W_{21}
\end{array}\right]\left[\begin{array}{ll|l}
V_{11}^{*} & 0 & V_{21}^{*}
\end{array}\right]} \\
& +\left[\begin{array}{cc}
0 & \gamma^{-1} W_{12} \\
I & 0 \\
\hline 0 & W_{22}
\end{array}\right]\left[\begin{array}{cc}
A_{\theta} & B_{\theta} \\
C_{\theta} & D_{\theta}
\end{array}\right]\left[\begin{array}{cc|c}
0 & I & 0 \\
V_{12}^{*} & 0 & V_{22}^{*}
\end{array}\right] \\
& \left(A \in C^{n \times n}, n=n_{0}+n_{\theta}\right)
\end{aligned}
$$

と書ける13). 補題 2 から, (27) 式の $(A, B, C, D)$ に対 ᄂ, $\left[\begin{array}{ll}C_{2} & D_{2}\end{array}\right]=\left[\begin{array}{ll}C_{\theta 2} & D_{\theta 2}\end{array}\right]\left[\begin{array}{ccc}0 & I & 0 \\ V_{12}^{*} & 0 & V_{22}^{*}\end{array}\right], Q$ が存在し て $(8),(13)$ 式が成り立ち, $(A, \bar{C})$ は可観測となる. (13) 式 $\Rightarrow(6)$ 式だから, 補題 1 より, (24) 式の $H(\zeta)$ が $(2)$, (3) 式を満たすことがいえた.

$(2),(3)$ 式を満たす $H(\zeta)$ が $(24)$ 式で表されること：補 題 1 より, $(2),(3)$ 式を満たす $H(\zeta)$ の適当な実現 $(A, B$, $C, D)\left(A \in C^{n \times n}\right)$ に対し, $C_{2}, D_{2}, Q$ が存在して (6), (8) 式が成り立ち, $(A, \bar{C})$ は可観測となる。いま,これらに 加えて $F_{2}=0$ となる場合を考える $\left(F_{2} \neq 0\right.$ の場合は後に 考える).このとき, 補題 $\mathbf{2}$ より, $(A, B, \bar{C}, \bar{D})$ は $(18)$ 式 の $\left(A_{0}, B_{0}, \bar{C}_{0}=\left[\begin{array}{c}\gamma^{-1} C_{0} \\ C_{02}\end{array}\right], \bar{D}_{0}=\left[\begin{array}{c}\gamma^{-1} D_{0} \\ D_{02}\end{array}\right]\right)$ の相似なシ ステムとして与えられる。この $\left(A_{0}, B_{0}, C_{0}, D_{0}\right)$ は $(27)$ 式の $(A, B, C, D)$ に等しく, これは $(24)$ 式の右辺の実現 の一つだったから，(2), (3) 式を満たす $H(\zeta)$ が $(24)$ 式 で表されることがいえた。つぎに， $F_{2} \neq 0$ の場合を考え る.このとき, $(A, B, C, D)$ に不可観測な部分システムを 付加して $H(\zeta)$ の新しい実現を作れば， $F_{2}=0$ の場合に 帰着できる. 以下にこれを示す。不可観測な部分システ ムを含む $H(\zeta)$ の新しい実現

$$
A^{\prime}=\left[\begin{array}{cc}
A & 0 \\
A_{3} & A_{4}
\end{array}\right], B^{\prime}=\left[\begin{array}{c}
B \\
B_{3}
\end{array}\right], C^{\prime}=\left[\begin{array}{ll}
C & 0
\end{array}\right], D^{\prime}=D
$$

を考える.さらに，この実現に対して

$$
C_{2}^{\prime}=\left[\begin{array}{ll}
C_{3} & C_{4}
\end{array}\right], \quad D_{2}^{\prime}=D_{3}
$$

とおく.ここで, $A_{3}, A_{4}, B_{3}, C_{3}, C_{4}, D_{3}$ は

$$
\begin{aligned}
& {\left[\begin{array}{ll}
A_{3} & B_{3} \\
C_{3} & D_{3}
\end{array}\right]=\left[\begin{array}{l}
A_{5} \\
C_{5}
\end{array}\right]\left[\begin{array}{ll}
C_{2} & D_{2}
\end{array}\right]} \\
& {\left[\begin{array}{ll}
A_{4} & A_{5} \\
C_{4} & C_{5}
\end{array}\right]\left[\begin{array}{c}
Q_{2} \Lambda \\
F_{2}
\end{array}\right]=\left[\begin{array}{c}
Q_{2} \\
0
\end{array}\right]} \\
& {\left[\begin{array}{ll}
A_{4} & A_{5} \\
C_{4} & C_{5}
\end{array}\right]^{*}\left[\begin{array}{ll}
A_{4} & A_{5} \\
C_{4} & C_{5}
\end{array}\right]=I}
\end{aligned}
$$


により定義される。この定義の妥当性を確認する。(29), (30) 式は (8), (13) 式と同様の関係であり, Lyapunov方 程式 $\Lambda^{*} P^{\prime} \Lambda-P^{\prime}+F_{2}^{*} F_{2}=0$ の解は $P^{\prime} \geq 0$ である. よっ て, 補題 2 より, (29), (30) 式を満たす $A_{4}, A_{5}, C_{4}, C_{5}$ が存在する. $A_{3}, B_{3}, C_{3}, D_{3}$ は $(28)$ 式から求められるか ら，(28) (30) 式は正しい定義式である.なお, 補題 2 より， $\left(A_{4}, C_{4}\right)$ が可観測であると仮定できる.そこで， $\bar{C}^{\prime}=\left[\begin{array}{c}\gamma^{-1} C^{\prime} \\ C_{2}^{\prime}\end{array}\right], \bar{D}^{\prime}=\left[\begin{array}{c}\gamma^{-1} D^{\prime} \\ D_{2}^{\prime}\end{array}\right]$ とおけば, $(6),(8),(9)$, (28)〜(30) 式などから

$$
\begin{aligned}
& {\left[\begin{array}{cc}
A^{\prime} & B^{\prime} \\
\bar{C}^{\prime} & \bar{D}^{\prime}
\end{array}\right]\left[\begin{array}{c}
Q^{\prime} \Lambda \\
E
\end{array}\right]=\left[\begin{array}{c}
Q^{\prime} \\
\bar{F}_{0}
\end{array}\right], \quad Q^{\prime}=\left[\begin{array}{c}
Q \\
Q_{2}
\end{array}\right]} \\
& {\left[\begin{array}{cc}
A^{\prime} & B^{\prime} \\
\bar{C}^{\prime} & \bar{D}^{\prime}
\end{array}\right]^{*}\left[\begin{array}{cc}
A^{\prime} & B^{\prime} \\
\bar{C}^{\prime} & \bar{D}^{\prime}
\end{array}\right]=I}
\end{aligned}
$$

が成立する。また， $(A, \bar{C}),\left(A_{4}, C_{4}\right)$ は可観測だから $\left(A^{\prime}, \bar{C}^{\prime}\right)$ も可観測となる。 以上より, $\left(A^{\prime}, B^{\prime}, C^{\prime}, D^{\prime}\right)$ を あらためて $(A, B, C, D)\left(A \in C^{n \times n}\right)$ と書いて, $H(\zeta)$ の 新しい実現を考えると, $F_{2}=0$ の場合へと帰着されるこ とが示された.よって， $F_{2}=0$ のときの結果を適用して， $(2),(3)$ 式を満たす $H(\zeta)$ が $(24)$ 式で表されることがい えた。

(24) 式のパラメトリゼーションは $H(\zeta)$ の LFT (Linear Fractional Transformation) 表現となっている.この関 係はFig. 1 に示される.ここで

$$
\left[\begin{array}{l}
y \\
v
\end{array}\right]=\Sigma(\zeta)\left[\begin{array}{l}
u \\
w
\end{array}\right], \quad w=\Theta(\zeta) v
$$

である。

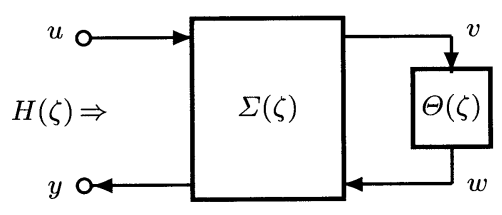

Fig. 1 Interpolating systems

最後に，いくつかの注意を記しておく.

（注意 1 ） 入出力特性 (1) 式が実軸対象に与えられたと する. すなわち, 複素数点 $\zeta_{i}$ が補間点ならば, $\zeta_{i}$ の共役複 素数点 $\zeta_{k}=\zeta_{i}^{*}$ も補間点であり, $q_{i}=q_{k}$ かつ $H_{i j}^{o}=H_{k j}^{o *}$ $\left(j=0,1, \ldots, q_{i}-1\right)$ とする. このとき, $(2),(3)$ 式を満た すすべてのシステムのパラメトリゼーションは, 実数值 演算だけにより，(24) 式で表現される実係数システムと して与えられる12).
（注意 2） $\nu=n_{0}+r$ または $\nu=n_{0}+m$ の場合を考え る. $\nu=n_{0}+r$ のとき, (15) 式の SVD は

$$
\left[\begin{array}{c}
Q_{0} \Lambda \\
E
\end{array}\right]=\left[\begin{array}{c}
V_{1} \\
V_{2}
\end{array}\right]\left[\begin{array}{ll}
\Sigma & 0
\end{array}\right]\left[\begin{array}{c}
U_{1}^{*} \\
U_{2}^{*}
\end{array}\right]
$$

と書け，また， $\nu=n_{0}+m$ のとき，(16) 式の SVDは

$$
\left[\begin{array}{c}
Q_{0} \\
\gamma^{-1} F
\end{array}\right]=\left[\begin{array}{l}
W_{1} \\
W_{2}
\end{array}\right]\left[\begin{array}{ll}
\Sigma & 0
\end{array}\right]\left[\begin{array}{c}
U_{1}^{*} \\
U_{2}^{*}
\end{array}\right]
$$

と書ける。これらの場合，(2), (3) 式を満たすシステムは 一意であり, その伝達関数はそれぞれ

$$
\begin{aligned}
& H(\zeta)= \Sigma_{1}(\zeta) \\
& \stackrel{\underline{S}}{=}\left\{\begin{array}{l|l}
{\left[W_{11} V_{1}^{*}\right.} & W_{11} V_{2}^{*} \\
\hline \gamma W_{21} V_{1}^{*} & \gamma W_{21} V_{2}^{*}
\end{array} \quad\left(\nu=n_{0}+r\right)\right. \\
& {\left[\begin{array}{l|l}
W_{1} V_{11}^{*} & W_{1} V_{21}^{*} \\
\hline \gamma W_{2} V_{11}^{*} & \gamma W_{2} V_{21}^{*}
\end{array}\right] \quad\left(\nu=n_{0}+m\right) }
\end{aligned}
$$

で与えられる.上式から， $\nu=n_{0}+r$ のとき，

$$
\gamma^{-2} H^{\sim}(\zeta) H(\zeta)=I_{r}
$$

となり,また， $\nu=n_{0}+m$ のとき，

$$
\gamma^{-2} H(\zeta) H^{\sim}(\zeta)=I_{m}
$$

となることがわかる．

（注意 3）補題 2 より，(27) 式の $(A, B)$ は, $\left(A_{\theta}, B_{\theta}\right)$ が可到達ならば, 可到達である。ところで, 定理 2 の証 明から，(8), (13) 式を満たす $(A, B, C, D)$ は一般に, 不 可観測な部分システムを含んでいることがわかる。した がって, $(27)$ 式の $(A, C)$ は, $\left(A_{\theta}, C_{\theta}\right)$ が可観測であっ ても, 必ずしも可観測でない. また, $\operatorname{deg} \Sigma(\zeta)=n_{0}$ であ る.これは $(25)$ 式の最右辺が $\Sigma(\zeta)$ の可到達・可観測な 実現を与えていることより確認できる。これらのことか ら，(24) 式において

$$
\begin{aligned}
\operatorname{deg} H(\zeta) & \leq \operatorname{deg} \Sigma(\zeta)+\operatorname{deg} \Theta(\zeta) \\
& =n_{0}+\operatorname{deg} \Theta(\zeta) \leq n
\end{aligned}
$$

となる。上式より，(2)，(3) 式を満たすシステムはたか だか $n_{0}$ 次で実現できる。実際には， $n_{0}$ 次より低次元の (2), (3) 式を満たすシステムが存在する。これは，(24) 式 において， $\Theta(\zeta)$ をうまく選んで不可観測な部分システム

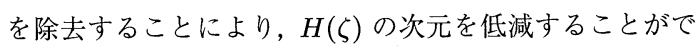
きるからである。

（注意 4）（2），(5) 式を満たすシステムが存在するため の必要十分条件は, Lyapunov 方程式 (12) の解 $P_{0}$ が 正定となることである. $P_{0}>0$ のとき, $(2),(5)$ 式を満 
たすすべてのシステムは $(24)$ 式でパラメトライズされ る.ただし, $\Theta(\zeta) \ll \gamma^{-1} \Theta(\zeta) \in B R H_{\infty}^{m \times r}$ なる任意の 関数である.このとき, $\Sigma_{21}(\zeta)$ は $r$ 次正方行列であり, $\operatorname{det} \Sigma_{21}(\zeta) \neq 0$ となることが確認できる.よって, (24) 式 の LFT 表現は次の HMT (Homogeneous Transformation）表現

$$
\begin{aligned}
H(\zeta)=\left\{\Pi_{11}(\zeta) \Theta(\zeta)+\Pi_{12}(\zeta)\right\} \\
\cdot\left\{\Pi_{21}(\zeta) \Theta(\zeta)+\Pi_{22}(\zeta)\right\}^{-1}
\end{aligned}
$$

へと変換できる13). 上式は参考文献 3),10) で与えられた (2)，(5) 式を満たすすべてのシステムのパラメトリゼー ションと同じ表現で斿る.すなわち，(24) 式のパラメト リゼーションは (3) 式に代えて (5) 式の条件を考えた場合 の結果を包含した表現となっている.

（注意 5）参考文献 9) では, 連続時間システムに対し, (2)，(5) 式を満たすすべてのシステムが (24) 式と同様の LFT 表現でパラメトライズされている。しかし，(2), (3) 式を満たすすべてのシステムのパラメトリゼーションは これと同様の簡潔な表現では与えられていない.

\section{4. 安定な補間システム}

ここでは, 3.の展開に基づいて, 問題 2 の解を示す. システムの伝達関数が $H(\zeta) \in R H_{\infty}^{m \times r}$ ならば, ある $\gamma>0$ が存在して $\gamma^{-l} H(\zeta) \in \bar{B} R H_{\infty}^{m \times r}$ が成り立ち, こ の逆もまた成立する。よって, 定理 1 より，(2), (4)式を 満たすシステムが存在するための必要十分条件は, ある $\gamma>0$ が存在して Lyapunov 方程式 (12) の解 $P_{0}$ が半正 定となることである。

ところで, $\Lambda$ は安定行列, $(\Lambda, E)$ は可観測だら, (12) 式で $\gamma \rightarrow \infty$ とした Lyapunov 方程式

$$
\Lambda^{*} \tilde{P}_{0} \Lambda-\tilde{P}_{0}+E^{*} E=0
$$

の解は $\tilde{P}_{0}>0$ である. (12) 式の解 $P_{0}$ の各成分は $\gamma$ に関 する連続関数だから， $\gamma$ を十分大きくとれば，(12)式の 解を必ず $P_{0} \geq 0$ とすることができる14).すなわち，(2), (4) 式を満たすシステムは必ず存在する.

上の考察から，(2): (4) 式を満たすすべてのシステム のパラメトリゼーションは, $\mathbf{3 .}$.展開を $\gamma \rightarrow \infty$ の場合 へと書き換えることにより導かれると理解できる．以下 にこの過程を展開する。

(31) 式の解 $\tilde{P}_{0}$ に対して, $\tilde{Q}_{0}=\tilde{P}_{0}^{\frac{1}{2}}$ とする.このと き, (15), (16) 式同様, 次の SVD が定義できる.

$$
\left[\begin{array}{c}
\tilde{Q}_{0} \Lambda \\
E
\end{array}\right]=\left[\begin{array}{cc}
\tilde{V}_{11} & \tilde{V}_{12} \\
\tilde{V}_{21} & \tilde{V}_{22}
\end{array}\right]\left[\begin{array}{c}
\tilde{\Sigma} \\
0
\end{array}\right] \tilde{U}^{*}
$$

$$
\tilde{Q}_{0}=\tilde{U} \tilde{\Sigma} \tilde{U}^{*}
$$

$$
\begin{aligned}
& \operatorname{rank}\left[\begin{array}{c}
\tilde{Q}_{0} \Lambda \\
E
\end{array}\right]=\operatorname{rank} \tilde{Q}_{0}=q r \\
& \tilde{\Sigma}=\operatorname{diag}\left[\begin{array}{lll}
\tilde{\sigma}_{1} & \cdots & \tilde{\sigma}_{q r}
\end{array}\right], \quad \tilde{\sigma}_{i}>0
\end{aligned}
$$

また,このSVDから, 次の行列を定義する.

$$
\tilde{W}=F \tilde{U} \tilde{\Sigma}^{-1}
$$

以上のもとで次のことがいえる.

【定理 3】（2)，(4)式を満たすすべてのシステムは

$$
H(\zeta)=\tilde{\Sigma}_{1}(\zeta)+\Xi(\zeta) \tilde{\Sigma}_{2}(\zeta)
$$

でパラメトライズされる.ここで, $\tilde{\Sigma}_{i}(\zeta)$ は

$$
\begin{aligned}
\tilde{\Sigma}(\zeta) & =\left[\begin{array}{c}
\tilde{\Sigma}_{1}(\zeta) \\
\tilde{\Sigma}_{2}(\zeta)
\end{array}\right] \\
& \stackrel{\mathrm{S}}{=}\left[\begin{array}{c|c}
\tilde{U} \tilde{V}_{11}^{*} & \tilde{U} \tilde{V}_{21}^{*} \\
\hline \tilde{W} \tilde{V}_{11}^{*} & \tilde{W} \tilde{V}_{21}^{*} \\
\tilde{V}_{12}^{*} & \tilde{V}_{22}^{*}
\end{array}\right]
\end{aligned}
$$

である.また, $\Xi(\zeta)$ は任意の $R H_{\infty}^{m \times r}$ の要素である. 証明に先だって, 補題 1 に対応する次の補題を示す.

【補題 3】次の二つの命題は等価である.

(i) $H(\zeta)$ は $(2),(4)$ 式を満たす.

(ii) $H(\zeta)$ の適当な実現 $(A, B, C, D)\left(A \in C^{n \times n}\right)$ に対 し, $\tilde{C} \in C^{r \times n}, \tilde{D} \in C^{r \times r}, \tilde{Q} \in C^{n \times q r}$ が存在して

$$
\begin{aligned}
& {\left[\begin{array}{cc}
A & B \\
\tilde{C} & \tilde{D}
\end{array}\right]^{*}\left[\begin{array}{cc}
A & B \\
\tilde{C} & \tilde{D}
\end{array}\right]=\left[\begin{array}{cc}
A & B \\
\tilde{C} & \tilde{D}
\end{array}\right]\left[\begin{array}{cc}
A & B \\
\tilde{C} & \tilde{D}
\end{array}\right]^{*}=I} \\
& {\left[\begin{array}{cc}
A & B \\
C & D
\end{array}\right]\left[\begin{array}{c}
\tilde{Q} \Lambda \\
E
\end{array}\right]=\left[\begin{array}{c}
\tilde{Q} \\
F
\end{array}\right]}
\end{aligned}
$$

が成り立ち, $(A, \tilde{C})$ は可観測となる。

(証明) (i) $\Rightarrow(\mathrm{ii})$ : 仮定より, $H(\zeta)$ の実現 $(A, B, C, D)$ ( $A$ : 安定行列, $(A, B)$ : 可到達) に対し, $\exists X>0 ; A X A^{*}+$ $B B^{*}=X$ となる. ここで, 適当な相似変換を施せば, 上 式は $A A^{*}+B B^{*}=I$ と書き換えられる（相似変換後も 同じ記法を用いた）。そこで，この $(A, B)$ に対し， $\tilde{C}, \tilde{D}$ が存在して (37) 式が成立する。このとき, $(A, \tilde{C})$ は可観 測となる。なぜなら, $(A, \tilde{C})$ が不可観測ならば, $\exists x \neq 0$; $A x=\lambda x(|\lambda|<1), \tilde{C} x=0$ となるが, これは (37) 式の $A^{*} A+\tilde{C}^{*} \tilde{C}=I$ に矛盾するからである. (2) 式が (38) 式 を含んでいることは，3.で述べたとおりである．

(ii) $\Rightarrow$ (i): 仮定より, 可観測な $(A, \tilde{C})$ に対して, $A^{*} A+$ $\tilde{C}^{*} \tilde{C}=I$ となるから, $A$ は安定行列である.ゆえに, $H(\zeta)$ は (4) 式を満たす. (37), (38) 式が $(2)$ 式を含んでいるこ とは，3.で述べたとおりである。 
(証明) (35) 式の $H(\zeta)$ が $(2),(4)$ 式を満たすこと： $\Xi(\zeta) \in R H_{\infty}^{m \times r}$ だから, 補題 $\mathbf{3}$ より $\Xi(\zeta)$ の適当な実現 $\left(A_{\xi}, B_{\xi}, C_{\xi}, D_{\xi}\right)\left(A_{\xi} \in C^{n_{\xi} \times n_{\xi}}\right)$ に対し, $\tilde{C}_{\xi}, \tilde{D}_{\xi}$ が存在 して

$$
\left[\begin{array}{cc}
A_{\xi} & B_{\xi} \\
\tilde{C}_{\xi} & \tilde{D}_{\xi}
\end{array}\right]^{*}\left[\begin{array}{cc}
A_{\xi} & B_{\xi} \\
\tilde{C}_{\xi} & \tilde{D}_{\xi}
\end{array}\right]=\left[\begin{array}{cc}
A_{\xi} & B_{\xi} \\
\tilde{C}_{\xi} & \tilde{D}_{\xi}
\end{array}\right]\left[\begin{array}{cc}
A_{\xi} & B_{\xi} \\
\tilde{C}_{\xi} & \tilde{D}_{\xi}
\end{array}\right]^{*}=I
$$

が成り立ち, $\left(A_{\xi}, \tilde{C}_{\xi}\right)$ は可観測となる。この $\left(A_{\xi}, B_{\xi}\right.$, $\left.C_{\xi}, D_{\xi}\right)$ を用いて, (35) 式の $H(\zeta)$ の実現の一つは

$$
\begin{aligned}
& {\left[\begin{array}{c|c}
A & B \\
\hline C & D
\end{array}\right]=\left[\begin{array}{c}
\tilde{U} \\
0 \\
\hline \tilde{W}
\end{array}\right]\left[\begin{array}{ll|l}
\tilde{V}_{11}^{*} & 0 & \tilde{V}_{21}^{*}
\end{array}\right]} \\
& +\left[\begin{array}{cc}
0 & 0 \\
A_{\xi} & B_{\xi} \\
\hline C_{\xi} & D_{\xi}
\end{array}\right]\left[\begin{array}{cc|c}
0 & I & 0 \\
\tilde{V}_{12}^{*} & 0 & \tilde{V}_{22}^{*}
\end{array}\right] \\
& \left(A \in C^{n \times n}, n=q r+n_{\xi}\right)
\end{aligned}
$$

と書ける. (40) 式の $(A, B, C, D)$ において, $\left[\begin{array}{ll}\tilde{C} & \tilde{D}\end{array}\right]=$ $\left[\begin{array}{ll}\tilde{C}_{\xi} & \tilde{D}_{\xi}\end{array}\right]\left[\begin{array}{ccc}0 & I & 0 \\ \tilde{V}_{12}^{*} & 0 & \tilde{V}_{22}^{*}\end{array}\right]$ ととれば，(37) 式が成立する. また， $\tilde{Q}=\left[\begin{array}{c}\tilde{Q}_{0} \\ 0\end{array}\right]$ ととれば, $(32) \sim(34)$ 式より，(38) 式 が成立する。ささらに，(A, $\tilde{C})$ が可観測であることは，補 題 $\mathbf{2}$ の証明と同様に確認できる。よって, 補題 $\mathbf{3}$ より, (35) 式の $H(\zeta)$ が $(2),(4)$ 式を満たすことがいえた.

$(2),(4)$ 式を満たす $H(\zeta)$ が $(35)$ 式で表されること：補 題 3 より，(2), (4) 式を満たす $H(\zeta)$ の適当な実現 $(A, B$, $C, D)\left(A \in C^{n \times n}\right)$ に対し, $\tilde{C}, \tilde{D}, \tilde{Q}$ が存在して (37), (38) 式が成り立ち， $(A, \tilde{C})$ は可観測となる。いま，これらに 加之て

$$
\left[\begin{array}{ll}
\tilde{C} & \tilde{D}
\end{array}\right]\left[\begin{array}{c}
\tilde{Q} \Lambda \\
E
\end{array}\right]=\tilde{F}=0
$$

となる場合だけを考えればよい。なぜなら， $\tilde{F} \neq 0$ の場 合, 定理 2 の証明の後半と同様に, $(A, B, C, D)$ に不可観 測な部分システムを付加して $H(\zeta)$ の新しい実現を作れ ば, $\tilde{F}=0$ の場合へと帰着されるからである. (37), (38), $(41)$ 式を満たし $(A, \tilde{C})$ が可観測な $\left(A, B,\left[\begin{array}{c}C \\ \tilde{C}\end{array}\right],\left[\begin{array}{c}D \\ \tilde{D}\end{array}\right]\right)$ $\left(A \in C^{n \times n}\right)$ は $n \geq q r$ でなければならず，そのような任 意の $\left(A, B,\left[\begin{array}{c}C \\ \tilde{C}\end{array}\right],\left[\begin{array}{c}D \\ \tilde{D}\end{array}\right]\right)$ は

$$
\begin{aligned}
{\left[\begin{array}{c|c}
A_{0} & B_{0} \\
\hline C_{0} & D_{0} \\
\tilde{C}_{0} & \tilde{D}_{0}
\end{array}\right]=} & {\left[\begin{array}{c}
\tilde{U} \\
0 \\
\hline \tilde{W} \\
0
\end{array}\right]\left[\begin{array}{cc|c}
\tilde{V}_{11}^{*} & 0 & \tilde{V}_{21}^{*}
\end{array}\right] } \\
& +\left[\begin{array}{cc}
0 & 0 \\
A_{\xi} & B_{\xi} \\
\hline C_{\xi} & D_{\xi} \\
\tilde{C}_{\xi} & \tilde{D}_{\xi}
\end{array}\right]\left[\begin{array}{cc|c}
0 & I & 0 \\
\tilde{V}_{12}^{*} & 0 & \tilde{V}_{22}^{*}
\end{array}\right]
\end{aligned}
$$

の相似なシステムとして与えられる。ここで, $\left(A_{\xi}, B_{\xi}\right.$,

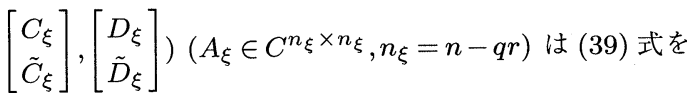
満たし $\left(A_{\xi}, \tilde{C}_{\xi}\right)$ が可観測な任意のシステムである。この ことは，(15) 式と (32) 式を，(16) 式と (33), (34) 式をそ れぞれ比較すれば， $\gamma \rightarrow \infty$ のとき， $V_{i j} \rightarrow \tilde{V}_{i j}, W_{11} \rightarrow$ $\tilde{U}, \gamma W_{21} \rightarrow \tilde{W}, W_{12} \rightarrow 0, W_{22} \rightarrow I$ となり, (18) 式右 辺 $\rightarrow(42)$ 式右辺となることから理解できる（撖密な証 明は省略する $).(42)$ 式の $\left(A_{0}, B_{0}, C_{0}, D_{0}\right)$ は $(40)$ 式の $(A, B, C, D)$ に等しく, これは $(35)$ 式の右辺の実現の一 つだったから，(2)，(4) 式を満たす $H(\zeta)$ が $(35)$ 式で表 されることがいえた。

（注意 6） (40) 式の $(A, B)$ は, $\left(A_{\xi}, B_{\xi}\right)$ が可到達なら ば，可到達である。このことは補題 $\mathbf{2}$ と同様に証明でき る.ところで, 定理 3 の証明から, (37), (38), (41) 式 を満たす $(A, B, C, D)$ は一般に, 不可観測な部分システ ムを含んでいることがわかる。よって，(40) 式の $(A, C)$ は, $\left(A_{\xi}, C_{\xi}\right)$ が可観測であっても, 必ずしも可観測でな い.また, (36) 式の最右辺は必ずしも $\tilde{\Sigma}(\zeta)$ の可観測な実 現を与えていない.これらのことから，(35) 式において

$$
\begin{aligned}
\operatorname{deg} H(\zeta) & \leq \operatorname{deg} \tilde{\Sigma}(\zeta)+\operatorname{deg} \Xi(\zeta) \\
& \leq q r+\operatorname{deg} \Xi(\zeta) \leq n
\end{aligned}
$$

となる，上式より，(2)，(4)式を満たすシステムはたかだ か $q r$ 次で実現できる。実際には, $q r$ 次より低次元の安 定な補間システムが存在する。これは，(35) 式において， $\Xi(\zeta)$ をうまく選んで不可観測な部分システムを除去す ることにより, $H(\zeta)$ の次元を低減することができるから である。

（注意 7） $\tilde{\Sigma}_{1}(\zeta)$ は $(2),(4)$ 式を満たす関数の一つであ り, $\tilde{\Sigma}_{2}(\zeta)$ は $(4)$ 式および

$$
\tilde{\Sigma}_{2}(\zeta)=O\left(\left(\zeta-\zeta_{i}\right)^{q_{i}}\right) \quad(i=1,2, \ldots, p)
$$

を満たす関数である。(35) 式はこれらの関数を用いて， (2), (4) 式を満たすすべてのシステムをパラメトライズ した式である。これは参考文献 3)で与えられた表現に一 致する，同参考文献では， $\tilde{\Sigma}_{1}(\zeta), \tilde{\Sigma}_{2}(\zeta)$ の具体的な構成 
手順には言及されていないが, 本稿では, これらの関数 を(36) 式によって与えている.

\section{5. 数值例}

これまでの理論を, 簡単な数值例を通して確認する.

【例題 1】入出力特性

$$
\begin{array}{ll}
p=2, & q_{1}=2, \quad q_{2}=1 \\
\zeta_{1}=0, & H_{10}^{o}=0, \quad H_{11}^{o}=\frac{1}{2} \\
\zeta_{2}=\frac{2}{3}, & H_{20}^{o}=\frac{1}{2}
\end{array}
$$

が与えられたとき, (3) 式を満たす補間システムを求める.

(12) 式の解 $P_{0}$ は

$$
P_{0}=\left[\begin{array}{ccc}
1 & 0 & 1 \\
0 & 1 & \frac{2}{3} \\
1 & \frac{2}{3} & \frac{9}{5}
\end{array}\right]-\gamma^{-2}\left[\begin{array}{ccc}
0 & 0 & 0 \\
0 & \frac{1}{4} & \frac{1}{4} \\
0 & \frac{1}{4} & \frac{9}{20}
\end{array}\right]
$$

となる.したがって, 定理 1 より, (3) 式を満たす補間シ ステムが存在するための必要十分条件は $P_{0} \geq 0$, すなわ ち, $\gamma \geq \gamma_{m}=(3+\sqrt{105}) / 16 \approx 0.8279$ で与えられる.

$\gamma=1$ のときを考える. このとき，(3) 式を満たすすべ ての補間システムは $(24)$ 式より

$$
H(\zeta)=\frac{\zeta\left(\frac{1}{2}+\frac{1}{7} \zeta\right)-\zeta^{2}\left(\frac{13}{42}-\zeta\right) \Theta(\zeta)}{\left(1-\frac{13}{42} \zeta\right)+\zeta\left(\frac{1}{7}+\frac{1}{2} \zeta\right) \Theta(\zeta)}
$$

$\Theta(\zeta):$ 任意の $\bar{B} R H_{\infty}$ の要素

と表される. $\left.\operatorname{rank} P_{0}\right|_{\gamma=1}=3$ だから，上式の補間シス テムはたかだか 3 次で実現できる（注意 $3 ）$. (43) 式よ り, $\Theta(\zeta)=$ 定数 $\neq 0$ ならば $\operatorname{deg} H(\zeta)=3, \Theta(\zeta)=0$ なら ば $\operatorname{deg} H(\zeta)=2$ である.また, $\Theta(\zeta)=(1 / 8) /(1-7 \zeta / 8)$ $\left(\|\Theta(\zeta)\|_{\infty}=1\right)$ ととれば

$$
H(\zeta)=\frac{\frac{1}{2} \zeta\left(1-\frac{2}{3} \zeta\right)}{\left(1-\frac{1}{2} \zeta\right)\left(1-\frac{2}{3} \zeta\right)}=\frac{\frac{1}{2} \zeta}{1-\frac{1}{2} \zeta}
$$

となるから, $\operatorname{deg} H(\zeta)=1\left(\|H(\zeta)\|_{\infty}=1\right)$ である.これ は (3) 式を満たす最小次数の補間システムとなる.

一方, $1>\gamma \geq \gamma_{m}$ のとき, (3) 式を満たす補間システ 厶の最小次数は 2 次である. とくに, $\gamma=\gamma_{m}$ のとき, 注 意 2 の条件が成り立つので, (3) 式を満たす補間システ ムは一意に定まる。そのシステムは

$$
H(\zeta)=\gamma_{m} \frac{\zeta\left(\frac{1}{2 \gamma_{m}}+\zeta\right)}{1+\frac{1}{2 \gamma_{m}} \zeta}
$$

で与えられ， $\gamma_{m}^{-2} H^{\sim}(\zeta) H(\zeta)=1$ が成立する.

【例題 2】入出力特性

$$
\begin{array}{ll}
p=3, & q_{1}=q_{2}=q_{3}=1 \\
\zeta_{1}=0, & H_{10}^{o}=1 \\
\zeta_{2}=\frac{1}{2}+j \frac{1}{2}, & H_{20}^{o}=\frac{1}{2}+j \frac{1}{2} \\
\zeta_{3}=\frac{1}{2}-j \frac{1}{2}, & H_{30}^{o}=\frac{1}{2}-j \frac{1}{2}
\end{array}
$$

が与えられたとき，(4) 式を満たす補間システムを求め る. 入出力特性は虚数を含んでいるが実軸対象に与えら れているので, 実数值演算によって実係数の補間システ ムを求めることができる（注意 1 ).

(35) 式より, (4) 式を満たすすべての補間システムは

$$
H(\zeta)=\frac{\left(1-\frac{5}{4} \zeta+\frac{3}{2} \zeta^{2}\right)-\zeta\left(\frac{1}{2}-\zeta+\zeta^{2}\right) \Xi(\zeta)}{1-\zeta+\frac{1}{2} \zeta^{2}}
$$

$$
\Xi(\zeta) \text { : 任意の } R H_{\infty} \text { の要素 }
$$

と表される.

(4) 式を満たす補間システムの最小次数は 2 次である. (4) 式を満たすすべての 2 次の補間システムは

$$
\begin{gathered}
\Xi(\zeta)=\frac{\left(\frac{3}{2}+2 \alpha_{1}+\alpha_{2}\right)-\left(1+\frac{1}{2} \alpha_{1}-\alpha_{2}\right) \zeta}{1+\alpha_{1} \zeta+\alpha_{2} \zeta^{2}} \\
\left(\left|\alpha_{1}\right|<1+\alpha_{2}, \quad\left|\alpha_{2}\right|<1\right)
\end{gathered}
$$

ととることにより

$$
H(\zeta)=\frac{1-\left(1+\frac{1}{2} \alpha_{2}\right) \zeta+\left(2+\alpha_{1}+\alpha_{2}\right) \zeta^{2}}{1+\alpha_{1} \zeta+\alpha_{2} \zeta^{2}}
$$

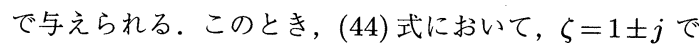
極零相殺が生じ, 次数の低減がなされている（注意 6 ).

一方, (4) 式の制約がなければ, 補間システムは 1 次で 実現できる。そのシステムは

$$
H(\zeta)=\frac{1-\zeta}{1-2 \zeta} \notin R H_{\infty}
$$

で与えられる。

\section{6. おわりに}

本稿では, 新しいアプローチに基づいて $H_{\infty}$ ノルム が $\gamma$ 以下であるすべての補間システムのパラメトリゼー ション, および, 安定なすべての補間システムのパラメ トリゼーションをそれぞれ与えた。

パラメトライズされた補間システムのうち, 最小次数 のシステムを求めることは未解決の問題である. 本稿のア プローチに基づけば，この問題はある制約のもとで Lya- 
punov 方程式の半正定解の階数を最小にする問題として 定式化できる15). その解法は今後の課題としたい.

\section{参 考文献}

1) R. E. Kalman: On minimal partial realizations of a linear input/output map; Aspects of Network and System Theory (R. E. Kalman and N. de Claris, Eds.), Holt, Reinhart and Winston, pp. 385-408 (1971)

2) A. J. Tether: Construction of minimal linear statevariable models from finite input-output data; IEEE Trans. Automatic Control, Vol. 15, No. 4, pp. 427436 (1970)

3) J. Ball, I. Gohberg and L. Rodman: Interpolation of Rational Matrix Functions; Operator Theory: Advances and Applications, Vol. 45, Birkhäuser Verlag, Part IV, V (1990)

4) A. C. Antoulas, J. A. Ball, J. Kang and J. C. Willems: On the solution of the minimal rational interpolation problem; Linear Algebra and Its Applications, Vol. 137/138, pp. 511-573 (1990)

5) 章, 細江, 伊藤 : 行列補間問題の Block-Reductionによる解 法 ; 計測自動制御学会論文集, Vol. 25, No. 2, pp. 159-166 (1989)

6) H. Kimura: Directional interpolation approach to $H^{\infty}$-optimization and robust stabilization; IEEE Trans. Automatic Control, Vol. 32, No. 12, pp. 10851093 (1987)

7) D. J. N. Limebeer and B. D. O. Anderson: An interpolation theory approach to $H^{\infty}$ controller degree bounds; Linear Algebra and Its Applications, Vol. 98, pp. 347-386 (1988)

8) H. Kimura: Directional interpolation in the state space; Systems 8 Control Letters, Vol. 10, pp. 317 324 (1988)
9) H. Kimura: Conjugation, interpolation and modelmatching in $H^{\infty}$; International Journal of Control, Vol. 49, No. 1, pp. 269-307 (1989)

10) K.-Z. Liu and T. Mita: Conjuation and $H^{\infty}$ control of discrete-time systems; International Journal of Control, Vol. 50, No. 4, pp. 1435-1460 (1989)

11) A. C. Antoulas and B. D. O. Anderson: On the problem of stable rational interpolation; Linear Algebra and Its Applications, Vol. 122/123/124, pp. 301-329 (1989)

12）堀口, 西村, 長田: 1 次・ 2 次情報を補間するシステムのパ ラメトリゼーション; システム制御情報学会論文誌, Vol. 7, No. 3, pp. 94-102 (1994)

13) M. Green and D. J. N. Limebeer: Linear Robust Control, Prentice-Hall, Chap. 3, 4 (1995)

14) 児玉, 須田：システム制御のためのマトリクス理論; 計測自 動制御学会, Chap. 13 (1978)

15) R. Amano, K. Horiguchi, T. Nishimura and A. Nagata: A parametrization of linear discrete-time systems interpolating input-output data; Proceedings of the 2nd Asian Control Conference, Vol. 1, pp. 493-496 (1997) 